\title{
Research and Development Trend of Shape Control for Cold Rolling Strip
}

\author{
Dong-Cheng Wang ${ }^{1,2} \cdot$ Hong-Min Liu ${ }^{1,2} \cdot J_{\text {Jun Liu }}^{1,3}$
}

Received: 17 November 2016/Revised: 4 May 2017/Accepted: 4 July 2017/Published online: 19 July 2017

(c) The Author(s) 2017. This article is an open access publication

\begin{abstract}
Shape is an important quality index of cold rolling strip. Up to now, many problems in the shape control domain have not been solved satisfactorily, and a review on the research progress in the shape control domain can help to seek new breakthrough directions. In the past 10 years, researches and applications of shape control models, shape control means, shape detection technology, and shape control system have achieved significant progress. In the aspect of shape control models, the researches in the past improve the accuracy, speed and robustness of the models. The intelligentization of shape control models should be strengthened in the future. In the aspect of the shape control means, the researches in the past focus on the roll optimization, mill type selection, process optimization, local strip shape control, edge drop control, and so on. In the future, more attention should be paid to the coordination control of both strip shape and other quality indexes, and the refinement of control objective should be strengthened. In the aspects of shape detection technology and shape control system, some new types of shape detection meters and shape control systems are developed and have successfully industrial applications. In the future, the standardization of shape detection technology and shape control system should be promoted to solve the problem of compatibility. In general,
\end{abstract}

Hong-Min Liu

liuhongmin_yanshan@126.com

1 National Engineering Research Center for Equipment and Technology of Cold Rolling Strip, Yanshan University, Qinhuangdao 066004, China

2 State Key Laboratory of Metastable Materials Science and Technology, Yanshan University, Qinhuangdao 066004, China

3 Anshan Iron and Steel Co. Ltd, Anshan 114002, China the four expected development trends of shape control for cold rolling strip in the future are intelligentization, coordination, refinement, and standardization. The proposed research provides new breakthrough directions for improving shape quality.

Keywords Cold rolling strip - Shape control · Development trend - Theory models - Shape detection . Control system

\section{Introduction}

With the serious excess of iron and steel production in the world, the profit of iron and steel enterprises is getting smaller and smaller, and the competition is more and more fierce. Continuous improvement of product quality has become one of the main measures to tide over the difficulties. In order to respond to the challenges, it is necessary to conduct a whole analysis of the present situation to seek new theories, technologies, measures and rules.

Cold rolling strip is widely used for automobiles, electrical appliances, building decoration, food packaging, electronic manufacturing, and so on. Strip shape is one important quality index of cold rolling strip. As a core high-end technology, shape control is not only the inevitable choice to produce high-grade cold rolling strip, but also the main requirement of production and equipment manufacturing upgrading in iron and steel industry.

Scholars have made profound and systematic researches in the shape control domain, achieving a wealth results. However, up to now, many problems in the shape control domain have not been solved satisfactorily, and a review on the research progress in the shape control domain can help to seek new breakthrough directions. 
Based on the above purpose, this paper makes a systematic investigation and analysis on the developments of shape theory models, shape control means, shape detection technology, and shape control system in the past 10 years. On the basis, the future development trends of shape control theory and technology are forecasted.

\section{Shape Control Models}

Shape control models include mechanism and intelligent models in terms of research methods. With regard to physical principles, shape control models consist of strip plastic deformation, rolls elastic deformation, strip and roll temperature field, rolls wear, strip buckling, shape pattern recognition, shape standard curve, and shape feedback control model [1]. Researches on shape control models have achieved remarkable progress in the past 10 years.

\subsection{Strip Plastic and Rolls Elastic Deformation}

The strip plastic and rolls elastic deformation models are the core of shape theory models, and they serve as the basis and premise of theory research on shape prediction and control. The modelling methods of strip plastic deformation include slab method, variation method, difference method, strip element method, and finite element method, while the modelling methods of rolls elastic deformation mainly include influence function method and finite element method. Research achievements in the strip plastic deformation model, rolls elastic deformation model, and the coupling method between them have further improved computational model accuracy, speed, and robustness.

Wang [2] proposed a new calculation method that combines the strip element method and engineering difference method to improve the accuracy and speed of strip plastic deformation model. Its calculation speed is high, and the prediction results of the strip profile and rolling pressure distribution are in good agreement with experimental and industrial data. Based on the difference method, Kong, et al. [3], proposed a fast algorithm using for rolls elastic deformation model, and the computation speed is improved by 1 time compared with that of the traditional method. Qin, et al. [4], proposed a matrix iteration method to improve the calculation speed of the rolls elastic deformation for a multi-rolls mill. A fast influence function method [5] using a high-order polynomial to describe the transverse distribution of rolling pressure and the pressure between rolls was proposed to significantly reduce the computation time. Zhu, et al. [6], Yang, et al. [7], built an integrated roll-and-strip shape prediction model that combined the $2 \mathrm{D}$ variable thickness finite element with the energy method or the 3D difference method, and its calculation speed is improved. Dixon, et al. [8], proposed a set of mechanism models of strip plastic and rolls elastic deformation, but the calculation procedure still requires iterative calculation between the calculation results of the two models, which affects its computational efficiency. Wang, et al. [9, 10], proposed a model coupling method to avoid the mutual iteration between the calculation results of strip plastic and rolls elastic deformation models, and its calculation speed is improved under the premise of ensuring calculation accuracy.

\subsection{Shape Pattern Recognition}

The shape pattern recognition model is a basic model of shape theory. It decomposes the deviation of the measuring shape and the target shape into a series of standard patterns, providing the basis for shape control. The traditional modelling method of shape pattern recognition is the leastsquares method based on Legendre or other orthogonal polynomials. The property that the integral of Legendre orthogonal polynomials along the strip width is zero satisfies the condition of the self-equilibrium of residual stress. Researches on the shape pattern recognition model mainly focuses on two aspects, namely, intelligence of the recognition algorithms and precision improvement of the standard patterns.

Recognition algorithms have developed rapidly. Various intelligent methods were adopted to improve anti interference capability [11-14]. Zhang, et al. [15], combined the genetic algorithm with the back propagation neural network to improve the capability and precision of shape recognition. The combination of the particle swarm optimization theory with the fuzzy method overcomes the shortcomings of traditional recognition methods [16]. The combination of the universal gravitation optimization algorithm and the support vector machine model improves model precision [17]. The cerebellar model used in shape pattern recognition reduces model training time [18]. Jia, et al. [19], improved the shape recognition precision significantly through combining the genetic algorithm, fuzzy theory, and neural network. Moreover, a series of novel shape pattern recognition models were established based on support vector machine, cloud model, neural network, genetic algorithm, and other intelligent methods combined with one another or with the traditional least-squares method [20-23].

In the aspect of the standard pattern, the traditional pattern generally involves linear, quadratic, and quartic orthogonal polynomials. Shan, et al. [24], proposed a new standard pattern containing the cubic component of strip shape. Bao, et al. [25], found that the cubic component of 
strip shape exists in the ultra-wide strip rolling process, and the cubic component of strip shape makes the results of shape pattern recognition highly accurate. Yang, et al. [26], believed that some complex shapes in the rolling process of an ultra-wide strip cannot be described by quartic orthogonal polynomials, thus, a vector extraction method of typical shape defects was proposed. Finally, the ratio of various typical defect vectors was obtained with a clustering algorithm.

\subsection{Strip Buckling}

The strip buckling model is a basic model of shape theory too. It is used to discriminate the state that the strip under a specific residual stress status is stable or not and to calculate the buckling deformation. Researches of strip buckling aim to improve the speed and accuracy of buckling and post-buckling models.

A reduced-order model of strip buckling was established based on the strip element discrimination method [27]. The number of unknowns is reduced by half through introducing the continuous two-order derivative of the nodal line and the stress boundary condition of the free edge, so its calculation speed has also improved significantly. Furthermore, the buckling and post-buckling models of the central wave and local shape defects were established by Zhang, et al. [28-31]. Theoretical and experimental studies on the buckling behavior of a strip with initial wave and a flat strip have been conducted to enrich the strip buckling theory. Nakhoul, et al. [32], applied the multi-scale method in solid mechanics to discriminate if the strip is unstable and to calculate the post-buckling behavior. Tran, et al. [33], studied the relation between residual stress, global tensile stress and buckling for wavy edge flatness defect during the mechanical unloading. Abdelkhalek, et al. [34], applied asymptotic numerical and finite element methods to predict the buckling and post-buckling behaviors of strips under any stress boundary condition, which is the most complete strip buckling model at so far.

\subsection{Shape Standard Curve}

The shape standard curve is also called shape target curve, which is the target of shape control. The traditional presetting method of shape standard curve is mainly based on experience and lacks in-depth theoretical analysis, which is the weakest part in shape theory models. Mechanism modeling of the shape standard curve has been promoted with the insights into the nature of the shape standard curve and the maturity of models in the shape prediction (strip plastic deformation theory) and shape discrimination (strip buckling theory).
The shape standard curve of the first stand of a tandem cold rolling mill was established by Bai, et al. [35] to control strip edge drop and keep the rolling process stable. The established curve compensates for the influence of the strip temperature transverse distribution, increases the bending force, and reduces the strip edge drop. The shape standard curve model was put forward for the high-strength steel according to the transverse distribution of strip temperature and the requirement of the subsequent processing [36], and the shape quality is improved. The strip shape standard curve in a tandem cold rolling mill was established to compensate for the comprehensive effects of the strip coiling shape variation, fetching machine installation error, strip transverse temperature difference, edge drop control, and other factors[37], and both the shape quality and the edge drop quality are improved. The possibility of the occurrence of holes and broken strips is reduced by dynamically setting the shape standard curve [38].

The complete mechanism method of the shape standard curve has been proposed by Liu, et al. [1]. The method combines shape prediction and discrimination models and considers the influence of the incoming crown, control requirement of the strip transverse thickness difference, and other factors. Its principles are to improve the strip crown to the maximum degree and ensure good shape (maintaining strip stability) in the first several passes while keeping the strip shape ideal in the last two passes. The method not only overcomes the drawback of the empirical method but also ensures comprehensive optimal control of strip shape and crown.

\subsection{Shape Feedback Control}

The shape feedback control model is used for closed-loop control. This model establishes the increment relationship between the shape control means and the shape parameters. A one-to-one mapping relation between the shape control means and the standard patterns is adopted by the traditional shape feedback control model. The interaction of various shape control means is not considered, showing that the theory is imperfect and its calculation precision is poor. Researches on the shape feedback control model aim to improve calculation accuracy and speed. According to the criterion whether the shape pattern recognition is used, the shape feedback control model can display in two forms, namely, multi-point control method and standard pattern control method.

According to the concept of the control efficiency coefficient, Wang, et al. [39], established a multi-point control model based on the increment relationship between the control means and the shape parameters of every point along the strip width, and designed an effective selflearning algorithm of the control efficiency coefficients, 
which improves the control precision of strip shape. Further more, the matrix analytical method was applied to realize shape control optimization under saturation statuses of single or multiple adjustment parameters [40]. The multi-point control method deals with the interaction of various shape control means and overcomes the deficiency of the traditional shape feedback control model. However, its calculation amount is large that its speed is low because of the use of numerous shape parameters along the strip width.

According to the concept of the influence function, the control matrix model was established based on the increment relationship between the control means and the shape standard patterns. Two calculation methods, namely, mechanism control matrix and intelligent control matrix, were also proposed to improve the reliability and versatility of models [41-43]. This standard pattern control method overcomes the shortcomings of the traditional shape feedback control model too. Its speed is fast because the method employs less shape parameters to represent the entire shape along the strip width.

\subsection{Shape Mechanisms Explored in Depth}

The presence of numerous influencing factors makes the generation mechanism of strip shape highly complex. Consequently, this mechanism requires extensive researches. Several new shape mechanisms have been established in recent years because of the progress in research methods, computing power, and experimental techniques. Abdelkhalek, et al. [44], analyzed the influence mechanism of the local shape instability at the exit of deformation zone on shape in detail, and a calculation method was put forward to solve the coupling relationship among strip plastic deformation, rolls elastic deformation, and strip buckling models, which improves the shape prediction precision. Kim, et al. [45], pointed out that the pre-deformation at the entry of deformation zone exerts a significant effect on strip shape, and the finite element simulation results are close to the calculation results in consideration of the effect of pre-deformation. Through finite element softwares, Abdelkhalek, et al. [44], Moazeni, et al. [46], proved again the conclusion[47] that the transverse distribution of strip flow velocity at the exit of deformation zone is unevenly and must be considered when the transverse distribution of tension stress is calculated. The industrial data collected by Wang, et al. [48], showed that the strip tension feedback mechanism exerts a significant effect on strip shape surely. Liu [49] identified the mechanisms of self-healing, dominant inheritance, and recessive inheritance of the incoming shape defects, and provided a method to quantitative calculating the influence of the incoming shape defects on the shape at the exit of deformation zone. Kapil, et al. [50],
Sun, et al. [51, 52] explored the dynamic characteristic of shape during the cold rolling process.

\subsection{Summary}

In the past 10 years, the researches of shape control models improve their accuracy, speed and robustness. In addition, some new shape mechanisms are explored. The breakthroughs of some new technology such as artificial intelligence and big data provide new hope for the development of shape control models. In the future, the intelligentization of the models should be strengthened.

\section{Shape Control Means}

The basic shape control means of cold rolling strip mainly include roll tilting, roll bending, roll shifting, roll cooling, roll profiles optimization, rolling process optimization, and so on. Researches on the basic shape control means, such as roll profile optimization, rolling mill type selection, rolling process optimization, local shape control, edge drop control, and comprehensive control of shape and surface quality, have achieved great progress in the past 10 years.

\subsection{Roll Profile Optimization}

The technology of roll profile design and optimization has been applied early and remains an important part of related research. The application range of roll profile optimization has expanded from simply improving strip shape to comprehensively controlling strip shape, crown, edge drop, and roll wear. In particular, edge drop control by optimizing the roll profile has become a popular research topic.

Taking the ideal shape as target, the back-up and work roll profiles of a temper rolling mill [53] and a variable crown rolling mill [54] were optimized, and roll-bending control efficiency and strip quality were improved. Taking the ideal shape and the uniformity of the pressure distribution among rolls as dual target, Bai, et al. [55], Xie, et al. [56], optimized back-up and work roll profiles to improve the shape quality, and the service life of rolls was enhanced. Yang, et al. [57, 58], achieved comprehensive control of strip shape, crown, and edge drop by optimizing the configuration of the work and back-up roll profiles.

\subsection{Rolling Mill Type Selection}

The shape control capability of a particular type of rolling mill exerts a decisive effect on strip quality. According to product positioning, analyzing and comparing the control 
performances of various rolling mill types, and selecting the appropriate rolling mill type are an essential task for newly built and rebuilt mills. Researches on rolling mill type selection aim to the scientificity of evaluation method and integrity of evaluation indexes.

A vector evaluation method for overall control of the whole crown and center crown was proposed by Peng, et al. [59]. The method can be used to analyze the advantages and disadvantages of 6-high HC, 6-high CVC, 4-high CVC, and ordinary 4-high mills and thus serves as an analytical tool and an important reference for rolling mill type selection. Xv[60] proposed a set of complete evaluation indexs system for shape control performance. The seven specific indexes are the adjustment range of the roll gap crown, roll gap transverse stiffness, uniformity of contact pressure among rolls, crown control performance of roll bending, edge drop control performance, shape controlling actuator efficiency, and the basic crown of loaded roll gap. This set of index system provides the measure for the comprehensive performance evaluation of various rolling mills. Liu [61] introduced two new indexes including the adjustment range of shape and shape control performance of roll bending, which make the indexs system more scientific and practical.

\subsection{Rolling Process Optimization}

Reasonable design of rolling process parameters is the premise to ensure shape quality. Improving strip shape through optimizing the rolling process is a traditional technical method. However, numerous new applications of this method have been established. Researches on rolling process optimization mainly focus on the diversification of optimization parameters and optimization targets.

Shape quality was improved by optimizing the emulsion flux of the process cooling and multi-zone cooling [62]. The steel-sticking phenomenon caused by the bad shape of the strip head was avoided by optimizing the roll-bending force [63]. Shape control difficulty was reduced by optimizing the strip elongation rate of the temper mill [64]. The shape quality, mechanical properties, and surface roughness of a strip rolled by a cold rolling mill were guaranteed by optimizing the rolling force and tension [65]. Comprehensive improvement of strip shape and surface quality could also be realized by optimizing the rolling force, strip elongation rate, and tension [66]. Under the premise of ensuring good strip shape, the flux, concentration, and temperature of the emulsion were optimized to prevent slipping and thermal scratching between the strip and rolls [67]. As a result, the cleaning degree of the strip surface is improved, and the emulsion consumption is reduced.

\subsection{Local Shape Control}

The local shape control is difficult because its scope is small. "Convex rib" is a typical local shape defect. It has become the focus of production enterprises of cold rolling strips in recent years. Convex rib is due to the existence of a local high point along the strip width, forming an apparent bulge corresponding to the position of the local high point. This defect leads to longitudinal convex rib at the local position after unwinding the coil and thus seriously affects product quality. Researches on this area have achieved significant progress in modelling the convex rib degree and prevention measures.

A set of mechanism model to predict the convex rib degree was proposed by Bai, et al. [68], and its calculation results are in good agreement with the measured values, which provides a theoretical basis and an analytical tool for the prevention of the convex rib defect. A large amount of industrial data collected by $\mathrm{Li}$, et al. [69], showed that the convex rib of cold rolling strip is mainly caused by the inheritance of hot rolling strip local high points, which pointes out the origin of preventing the convex rib. Zhu, et al. [70], established a mathematical model of the critical condition for the convex rib and found that appropriately reducing the tension and increasing the friction coefficient can effectively prevent the convex rib.

$\mathrm{Yu}$, et al. [71], put forward a roll profile optimization technology of hot rolling mill, which is effective to prevent the convex rib for cold rolling strip. The possibility of convex rib defect in a cold rolling strip was effectively reduced by reasonably arranging the hot rolling schedule, controlling the thermal crown of hot rolling work roll, and optimizing the roll profile [72]. Improving the uniformity of slab heating temperature, decreasing the bending force, and increasing the deformation of the last pass were also used to control the strip convex rib [73]. Lu, et al. [74], reduced the strip convex rib ratio through optimization process and implementing strict process standards of hot rolling, cold rolling, and continuous annealing.

\subsection{Edge Drop Control}

The edge drop control technology can reduce the cutting loss and increase the yield. The application of edge drop control technology to silicon steel has received increasing attention in recent years. The research achievements of edge drop control technology are mainly embodied in developing automatic control systems and designing roll profiles.

The most advanced edge drop control technology at present is the K-WRS technology of the 6-high UCMW cold rolling mill. This technology was successfully applied to a $1550 \mathrm{~mm}$ tandem cold rolling mill at Baosteel [75-78]. This development indicates that the edge drop control technology of the 6-high UCMW cold rolling mill has matured. 
However, most of the cold rolling mills are 6-high UCM rolling mills or 4-high CVC rolling mills. Given that the 4-high CVC mill has work roll shifting function, it can use the K-WRS technology to realize similar function of the 6-high UCMW mill [79]. However, the edge drop control capacity of the 6-high UCM rolling mill is obviously insufficient because of the lack of work roll shifting function. Sun, et al. [80], adopted a single-side straighttaper work roll profile to enhance the edge drop control capacity of the 6-high UCM rolling mill. He, et al. [81], designed a sextic polynomial work roll curve and achieved remarkable results.

\subsection{Comprehensive Control}

With the continuous increasing demand of high surface quality, the researches on the control of surface roughness, surface cleanliness, surface color difference, and so on, have emerged [82]. Optimizing the rolling process parameters is a common and effective technical measure to improve surface quality. In related researches, surface quality control is often combined with shape control.

Comprehensive control of the surface roughness and shape of a double cold reduction mill was realized by optimizing the process parameters, such as rolling force and tension [65]. The rolling force, strip elongation rate, and tension of temper rolling mill were optimized to realize comprehensive control of the surface quality and shape of temper rolling strip [66]. In addition, the surface cleanliness and shape of the strip were improved by optimizing the flux, concentration, and temperature of the emulsion [67]. At present, the applications of the comprehensive control technology are pretty rare and worthy to be further promoted.

\subsection{Summary}

In the past 10 years, the researches of the shape control means mainly focus on the roll optimization, mill type selection, process optimization, local strip shape control, edge drop control, and so on. However, the shape control objective and variable are usually single and rough, lack of global concept. In the future, more attention should be paid to the coordination control of both strip shape and other quality indexes, and the refinement of control objective should be strengthened.

\section{Shape Detection Technology}

Shape detection is the premise to realize shape closed-loop control and is the key to improve shape quality. The strip shape meter is the "eye" of online detection. It is a high- end measuring instrument of the rolling process. The strip shape meter consists of two main parts, namely, shape detection roller and shape signal transmission processor. The development of a shape meter is so difficult that it has been monopolized for a long time by Sweden's $A B B$, Germany's SIEMENS, SUNDWIG, and other international companies [83-87]. In the past 10 years, a major breakthrough on the shape meter has achieved in China. The seamless shape detection roller and wireless shape signal transmission processor have been independently developed and successfully applied in industry [88-91].

\subsection{Seamless Shape Detection Roller}

The internationally popular sectional shape detection roller is shown in Figure 1 [92, 93]. This roller consists of a core shaft, outer rings and piezo-magnetic sensors. The strip surrounds the shape detection roller to form a certain angle. Strip tension $T$ acts on the shape detection roller and results in pressure $N$. A series of outer rings and sensors are arranged along the axial direction of the shape detection roller, and pressure $N$ is transmitted and detected. The axial pressure distribution is converted into tension distribution by the signal-processing computer so that the strip shape can be calculated. This kind of shape detection roller may crush and scratch the strip surface for two reasons. First, a gap exists among the outer rings. Second, the axial temperature of the shape detection roller is different. The temperature difference between the meter roller's middle and edge is approximately dozens of degrees Celsius, resulting in radial thermal expansion differences among different outer rings.

An inlayed block shape detection roller, which is shown in Figure 2, was developed by Yanshan University to overcome the shortcomings of sectional shape detection roller [94]. Two rectangular grooves are machined on the body of the detection roller, and a set of elastic blocks with sensors is installed in each rectangular groove. The structure effectively avoids scratching the strip surface caused by the uneven thermal expansion of the sectional detection roller. However, the "skin effect" of the current easily leads to uneven hardness of the rectangular groove edge and other part of the roller body when heat treatment is applied. The broken strip can easily damage the soft part of

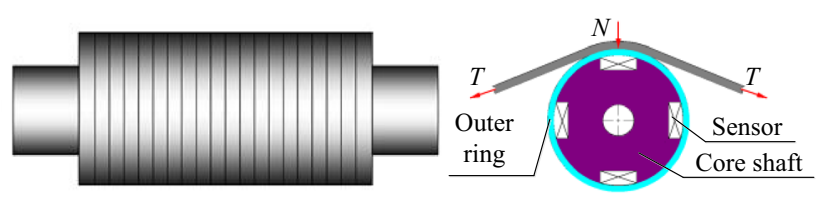

Figure 1 Sectional shape detection roller 


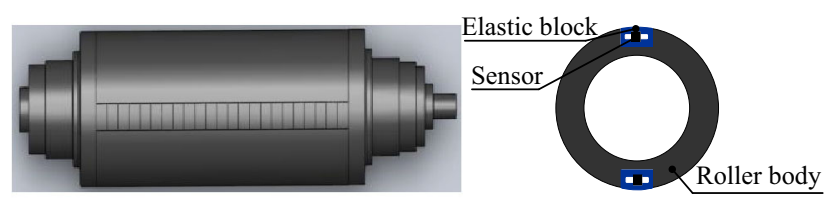

Figure 2 Inlayed block shape detection roller

the roller body, and the damaged roller surface may scratch the strip surface in subsequent production.

A new type of seamless shape detection roller was developed to completely solve the mentioned problems [95]. This shape detection roller is shown in Figure 3. Two to four precise through-holes are machined along the circumferential direction near the roller surface in the roller body. The wall thickness between the hole and the outer surface of the roller is between $6 \mathrm{~mm}$ and $8 \mathrm{~mm}$. The wall thickness must not only be conducive to the pressure transfer, but also retain enough thickness for the roller grinding. A series of sensors are arranged inside each through-hole. The roller material is GCr15, and the roller surface hardness exceeds $60 \mathrm{HRC}$ after quenching. The hardened layer thickness is $4 \mathrm{~mm}$ or more. This shape detection roller presents the advantages of seamless surface, high hardness, and deep hardened layer, which completely solve the technical problems of crushing and scratching strip surface.

A certain pre-pressure is applied on the sensor by an interference fit manner to keep the sensor working in the linearity range [96]. In fact, a certain temperature difference and thermal deformation difference exist between the outer surface of the detection roller and the internal sensor in the rolling process, and these differences may weaken or reduce the magnitude of interference and pre-pressure between sensors and the inner wall of through-holes, resulting in detection signal distortion. Therefore, the assembly of sensors must have an adequate magnitude of interference and pre-pressure. To solve the problem, the temperature field, thermal deformation, and pressure transfer of the detection roller and sensors in the rolling process were simulated with the finite element software. The structure size of the roller and the magnitude of interference were optimized according to the simulation results [97, 98].

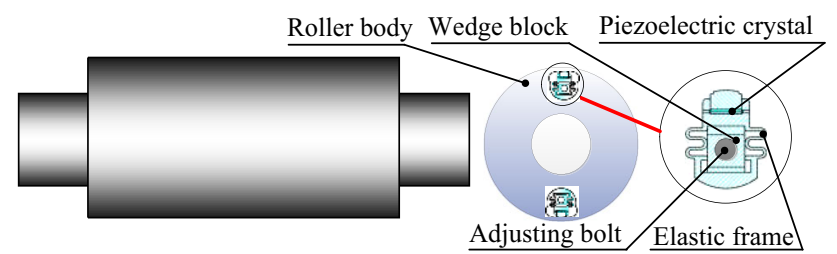

Figure 3 Seamless shape detection roller

\subsection{Wireless Signal Transmission Processor}

The carbon brush and slip ring are the popular structures for signal transmission and power supply, as shown in Figure 4. A series of copper slip rings are fixed on the rotating end of the detection roller's neck, and a series of carbon brushes are fixed on the cover that is connected with the bearing chock. The signals of the detection roller are transmitted to the slip rings via wires. The rotating slip rings then transmit the signals to the fixed carbon brushes. Finally, the signals are transmitted to the remote terminals via long-distance wires. The principle of power supply is similar with that of signal transmission. Owing to the friction between the carbon brush and slip ring, wear, vibration, and serious signal distortion may occur. The carbon brush and slip ring needed to be maintained frequently and equipped with cooling and cleaning devices. The remote transmission of analog signal can be easily interfered by electromagnetic, vibration, temperature, and other factors, which may increase the error.

A wireless and integrated signal transmission processor, as shown in Figure 5, was developed by Yanshan University to solve the above problems [99]. The signal processor consists of a rotating head and a fixed cover. The rotating head is connected to the detection roller and rotates

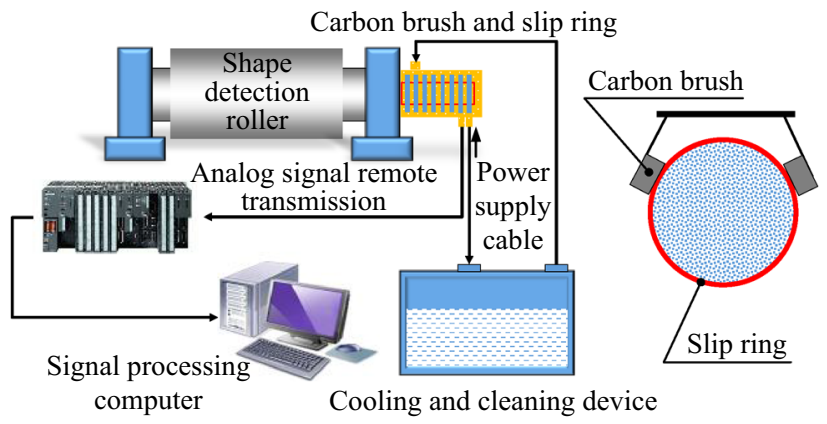

Figure 4 Carbon brush and slip ring structure

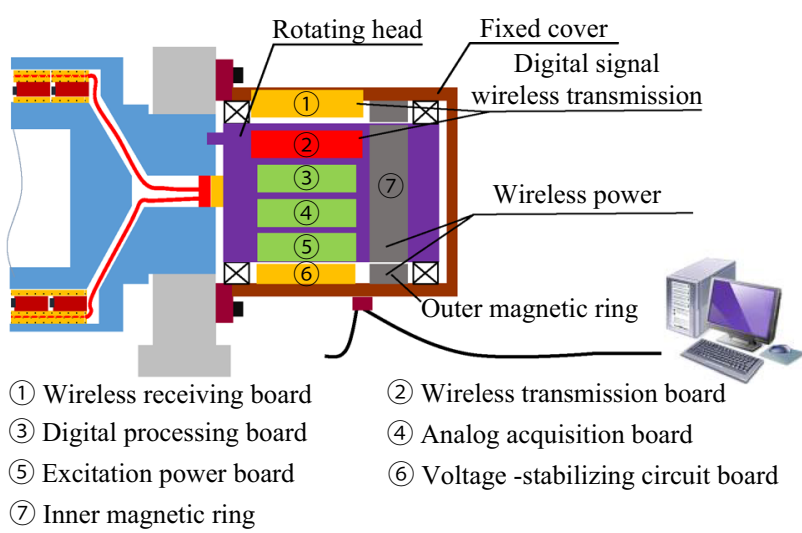

Figure 5 Wireless signal processing and power supply system 
synchronously. An excitation power board, an analog acquisition board, a digital processing board, a wireless transmission board, and an inner magnetic ring are arranged on the rotating head. The cover is fixed on the bearing chock, and a wireless receiving board, an outer magnetic ring, and a voltage-stabilizing circuit board are installed on the cover.

The working principle is that power is supplied to the wireless receiving board and the outer magnetic ring on the cover by the cable and voltage-stabilizing circuit board. The rotating inner magnetic ring receives the voltage-stabilizing signal by wireless induction transmission, and all sensors in the detection roller and all the circuit boards on the rotating head are powered by the excitation power board. The output signals of sensor are gathered by the analog acquisition board, and the digital processing board implements analog-to-digital conversion. Digital processing and coding are performed under the conditions of magnetic coupling isolation and photoelectric isolation. The signals are then delivered to the wireless transmission board. The wireless transmission board converts the signals into high-frequency wireless signals and transmits the wireless signals to the wireless receiving board. The wireless receiving board converts the wireless signals to wired signals. Finally, the signals are transmitted to the remote signal-processing computer via the shielded cable.

\subsection{Signal Denoising and Error Compensation}

Shape detection is affected by vibration, electromagnetic, temperature difference, detection roller installation error, deformation, coil shape changes, and other factors, so the shape detection signals need noise cancellation and error compensation to ensure the precision.

A discrete-time tracking differentiator was applied to eliminate the noise of the shape detection signal by $\mathrm{He}$, et al. [100]. Wavelet denoising technology was used to smooth the detection signal by Shan, et al. [101]. Li, et al. [102, 103], analyzed the influence of installation error and deformation of detection roller on the shape signal and established the compensation models, finally, the installation accuracy standards of shape detection roller was developed. A compensation model for installation error of shape detection roller was provided under the condition of no guide roller [104]. Yang, et al. [105], analyzed the influence of zero residual voltage, coverage width of the detection unit, installation errors and deformation of detection roller, and other factors on the shape detection signal, and a relatively complete compensation model was established. Wang, et al. [106], developed a compensation model for partial coverage of the strip edge and proposed an interpolation method for the fault measurement unit. All these studies have improved the accuracy of shape detection.

\section{Shape Control System}

The shape control system can be divided into pre-setting control system and closed-loop control system according to the control sequence, and the modelling of shape control system includes mechanism and intelligent models. Presetting control is a predictive control, and closed-loop control is a monitoring control. The two control systems complement each other and ensure the strip shape quality. The mechanism models are based on solid theoretical basis and have strong expansibility, but it is hard to realize accurate modelling and fast calculation. The intelligent models demonstrate strong learning capability, fast calculation speed, and simple modeling but poor analyticity and scalability. Coordination between these two models can effectively improve the control level. The shape control system of cold rolling strip is a complex control system with multiple variables, strong coupling, and nonlinearity. For a long time, the whole set of shape control system can only be provided by German's SIEMENS, Japan's MITSUBISHI, or other large international companies. In the past 10 years, several new computer control systems are developed for the cold rolling strip. Its industrial applications have achieved good results [107-109].

\subsection{Shape Pre-Setting Control}

Shape pre-setting control is the foundation of closed-loop control. Closed-loop control precision, speed, and stability are directly influenced by the precision of pre-setting control. Research progress in shape pre-setting control is mainly manifested in the aspects of improving calculation accuracy and speed.

Gong, et al. [110], established a shape pre-setting control model of a 6-high reversing cold rolling mill based on the data regression method. Another regression model of shape pre-setting control for the 6-high reversing cold rolling mill was established based on the results of the influence function method [111]. The regression models possess the advantages of simple structure and high calculation speed. Based on the mechanism model, Bai, et al. [112], divided shape pre-setting course into two steps, namely rough calculation step and precise calculation step, and a set of optimization algorithm was proposed to improve calculation speed. Wang [2] developed the models and softwares of shape pre-setting control for cold rolling strip according to the basic model of shape theory, which was successfully applied to multiple sets of cold rolling mills. The models and softwares are characterized by the strip element method. Under the premise of ensuring model accuracy, the calculation speed is improved significantly by the multistep optimization strategy. The presetting calculation time is less than $1 \mathrm{~s}$. 


\subsection{Shape Closed-Loop Control}

The core and key in shape closed-loop control are the control models, which include the control strategy and model algorithm. When the strip shape signal is detected, the control model rapidly calculates the adjustment amount of control means (e.g., roll tilting amount, roll bending force, multi-zone cooling spray) to realize timely and accurate adjustment of the strip shape. Research progress in the model of the closed-loop control is mainly embodied in the aspects of matrix model considering the mutual influence of various shape control means, collaboration technology between mechanism and intelligence models, and dynamic self-adaptive technology.

Jelali [113] introduced the monitoring technology to shape closed-loop control system of the cold rolling strip. The insufficiency of control system was observed and the adaptive improvement was realized through the online batch evaluation and shock diagnosis. Benporad, et al. [114], proposed three different control strategies based on quadratic optimization and hysteresis compensation techniques and significantly improved the performance of control system. Pin, et al. [115, 116], decomposed the shape signal into several orthogonal components corresponding to different shape control means, and the adjustment amount of control means was obtained by constrained optimization. The control precision was then improved by online adaptive technology. A control model of asymmetrical shape was established by evaluating the influence of roll tilting, work roll and intermediate roll asymmetrical bending on the asymmetrical shape through simulation calculation [117]. Furthermore, a reduced dimension control model and two times control strategies were proposed based on Legendre orthogonal polynomials and the concept of shape efficiency coefficient to improve the control performance [118].

Liu, et al. [41-43], proposed a shape control matrix model, a dynamic matrix model and a mechanism-intelligence collaboration matrix model in turn based on the relation between the shape control means and the shape standard patterns. The mechanism-intelligence collaboration control system is shown in Figure 6. The mechanism control matrix is established based on the calculation data of the mechanism models, and the intelligent control matrix is established based on the measured data on the rolling process. The two control schemes are weighted to form a final closed-loop control scheme. Control precision can be improved by the self-adaptive adjusting of the weight coefficient.

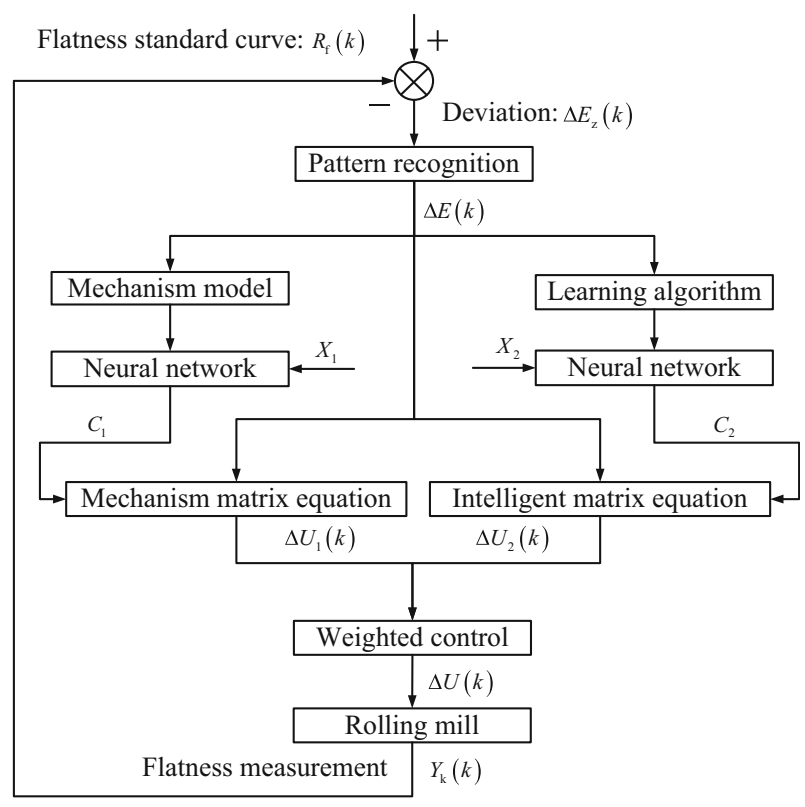

Figure 6 Mechanism-intelligence collaboration control system

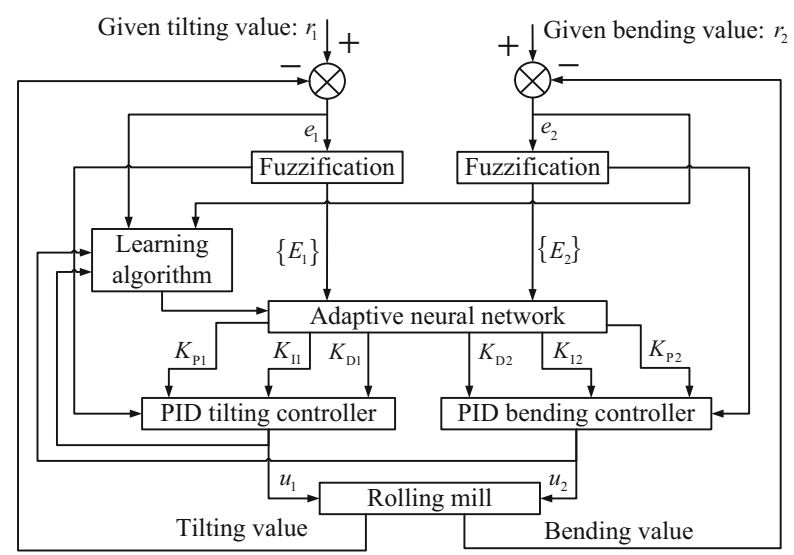

Figure 7 Fuzzy-neural PID model

\subsection{Intelligent Regulation of Tilting and Bending}

Roll tilting and bending are the most flexible and fast shape control means. The fuzzy-neural PID model of roll tilting and bending adjustment was established based on the coordination of the two intelligent methods of fuzzy theory and neural network [119]. The fuzzy-neural PID model is shown in Figure 7. The conventional PID control algorithm cann't be directly adapted to the complex and changeable rolling process, so the fuzzy theory, which is good at dealing with unknown models, is combined with the neural network with a strong self-learning capability to improve the shape control effect. 


\subsection{Application of Shape Detection and Control System}

The seamless and wireless shape meter and the mechanism-intelligence collaboration control system are successfully applied to $1780 \mathrm{~mm}$ temper rolling mill and 2130 $\mathrm{mm}$ skin pass mill in Ansteel. The strip quality is obviously improved from common level to advanced level. Such a development is of great significance for metallurgical industry technology upgrading and product quality improvement.

\subsection{Summary}

In the past 10 years, some new types of shape detection meters and shape control systems are developed and have successfully industrial applications, which provide more choices for the iron and steel plants. However, it also increases the compatibility difficulty. In the future, the standardization of shape detection technology and shape control system should be promoted to solve the problem of compatibility.

\section{Development Trend of Shape Control}

\subsection{Intelligentization}

The global manufacturing industry has opened the curtain for the fourth industrial revolution [120]. Germany has developed the "Industrial 4.0" program, the United States has put forward the "Advanced Manufacturing Partner" program, and China has formulated the "China Manufacturing 2025" plan. The iron and steel industry, which accounts for a large proportion of the manufacturing industry, is the key industry in the implementation of the above plans.

The intelligentization of shape control technology needs to be closely combined with the industrial internet of things, big data, artificial intelligence and cloud computing technologies.

\subsection{Coordination}

Strip shape, thickness, and surface quality are important quality indexes of cold rolling strip, and their control technologies are all the key technologies for cold rolling mill. The control systems of these indexes, which are both relatively independent and mutually influenced, must be coordinated handling to ensure the overall quality of the cold rolling strip. Therefore, the influence of all control means on the shape, thickness, and surface quality of the strip should be evaluated comprehensively.

\subsection{Refinement}

At present, the shape control technology can be easily using to eliminate single-edge, bilateral, and central waves. However, for some more complicated shape defects, such as edge-central wave, quarter wave, local wave, and edge drop, the control effect does not satisfy the requirement of industrial production. For these complicated shape problems, some more fine control technology, such as fine multi-zone cooling and fine roll profile optimization need to be further studied.

\subsection{Standardization}

A large number of researches and applications have resulted in abundant scientific experience and achievements in theoretical models, engineering software, shape control system designing, installation, commissioning, operation, and maintenance. However, the industry technical standards of shape control have not been drafted, which influences technology standardization and the capability to lead and promote the development of industry technology.

In the future, the technical standards of shape control need to be studied and drafted as soon as possible.

\section{Conclusions}

(1) Shape control is not only the inevitable choice to produce high-grade cold rolling strip, but also the main requirement of production and equipment manufacturing upgrading in iron and steel industry. In the past 10 years, researches and applications of shape control models, shape control means, shape detection technology, and shape control system have achieved significant progress.

(2) In the aspects of shape control models and shape control means, the intelligentization of shape control models should be strengthened. More attention should be paid to the coordination control of both strip shape and other quality indexes, and the refinement of control objective should be strengthened too.

(3) In the aspects of shape detection technology and shape control system, some new types of shape detection meters and shape control systems are developed and have successfully industrial applications. In the future, the standardization of shape detection technology and shape control system should be promoted to solve the problem of compatibility.

(4) In the future, the four expected development trends of shape control are intelligentization, coordination, refinement, and standardization. 
Acknowledgements Supported by National Key Technology Support Program of China (Grant No. 2011BAF15B00), Hebei Provincial Natural Science Foundation of China (Grant No. E2016203482), Hebei Provincial Science and Technology Research Foundation of China (Grant No. ZD2014034), and Youth Independent Research Program of Yanshan University of China (Grant No. 14LGA003).

Open Access This article is distributed under the terms of the Creative Commons Attribution 4.0 International License (http://crea tivecommons.org/licenses/by/4.0/), which permits unrestricted use, distribution, and reproduction in any medium, provided you give appropriate credit to the original author(s) and the source, provide a link to the Creative Commons license, and indicate if changes were made.

\section{References}

1. H M Liu, K R Ding, X D Li, et al. Theoretical computational method of shape standard curve. Chinese Journal of Mechanical Engineering, 2008, 44(8): 137-142. (in Chinese)

2. D C Wang. Research on theory models of online shape pre-set control for strip tandem mill. Qinhuangdao: Yanshan University, 2009. (in Chinese)

3. F F Kong, A R He, J Shao. Research on rapid online calculation methods of roll stack deformation. Chinese Journal of Mechanical Engineering, 2012, 48(2): 121-126. (in Chinese)

4. J Qin, Q Miao. The matrix iteration method for elastic deformation of multi-roll mill based on influence function method. Engineering Mechanics, 2013, 30(5): 271-276. (in Chinese)

5. D C Wang, Y L Wu, H M Liu. High-efficiency calculation method for roll stack elastic deformation of four-high mill. Iron and Steel, 2015, 50(11): 69-74. (in Chinese)

6. J R Zhu, X Z Lin, P Wu, et al. Development of unified simulation model of rolling for 6-Hi UCMW wide strip cold mill. Metallurgical Equipment, 2005, 8(4): 7-10, 28. (in Chinese)

7. Y T Yang, Q D Zhang, W G Wang, et al. Research on an integrative simulating model for strip shape prediction during cold rolling. Steel Rolling, 2007, 24(5): 13-16. (in Chinese)

8. A E Dixon, W Y D Yuen. A physical based method to predict spread and shape during flat rolling for real-time application. Steel Research International, 2008, 79(4): 287-296.

9. D C Wang, Z X Zhao, H M Liu. High-efficiency software and its application for shape analysis and presetting of cold rolling strip. Iron and Steel, 2015, 50(7): 54-60. (in Chinese)

10. D C Wang, H M Liu. A model coupling method for shape prediction. Journal of Iron and Steel International, 2012, 19(2): 22-27.

11. X W Zhang, Y Wang. Application and development trend of intelligent recognition methods for flatness recognition. Journal of Iron and Steel Research, 2010, 22(1): 1-13. (in Chinese)

12. H S Di, G M Liu, G W Jiang. A review of pattern recognition method for measured signals of shape in cold strip rolling. Henan Metallurgy, 2009, 17(4): 1-6, 24. (in Chinese)

13. C Q Huang, M Zhao. Research progress of plate-profile recognition and control for cold-rolled strip. Journal of Iron and Steel Research, 2013, 25(12): 2-7. (in Chinese)

14. L Hao, H S Di, D Y Gong, et al. Software development of strip flatness off-line display and pattern recognition. Journal of Northeastern University (Natural Science), 2010, 31(10): 1414-1416, 1420. (in Chinese)

15. C Zhang, J P Tan. Strip flatness pattern recognition based on genetic algorithms-back propagation model. Journal of Central South University (Science and Technology), 2006, 37(2): 294-299. (in Chinese)
16. J Liu, Y Q Wang, F Sun, et al. Fuzzy pattern recognition method if flatness based on particle swarm theory. Chinese Journal of Mechanical Engineering, 2008, 44(1): 173-178. (in Chinese)

17. P F Niu, P F Li, G Q Li, et al. Application of GSA-SVR model in flatness pattern recognition. Iron and Steel, 2012, 47(12): 45-49. (in Chinese)

18. H T He, Y Li. A new flatness pattern recognition model based on cerebellar model articulation controllers network. Journal of Iron and Steel International, 2008, 15(5): 32-36.

19. C Y Jia, X Y Shan, H M Liu, et al. Fuzzy neural model for flatness pattern recognition. Journal of Iron and Steel International, 2008, 15(6): 33-38.

20. X L Zhang, S Y Zhang, G Z Tan, et al. A novel method for flatness pattern recognition via least squares support vector regression. Journal of Iron and Steel International, 2012, 19(3): $25-30$.

21. X L Zhang, S Y Zhang, W B Zhao, et al. A novel method for flatness pattern recognition via MLSSVR. China Mechanical Engineering, 2013, 24(2): 258-263. (in Chinese)

22. X L Zhang, T Xv, L Zhao, et al. Method of flatness pattern recognition based on GA-PID neural network. Journal of Shenyang University (Natural Science), 2013, 25(3): 209-215. (in Chinese)

23. X L Zhang, L Zhao, J Y Zang, et al. Flatness intelligent based on T-S cloud inference neural network. ISIJ International, 2014, 54(11): 2608-2617.

24. X Y Shan, H M Liu, C Y Jia. A recognition method of flatness pattern containing the cubic flatness. Iron and Steel, 2010, 45(8): 56-60. (in Chinese)

25. R R Bao, J Zhang, H B Li, et al. Flatness pattern recognition of ultra-wide tandem cold rolling mill. Chinese Journal of Engineering, 2015, 37(S1): 6-11. (in Chinese)

26. G H Yang, J Zhang, H B Li, et al. Extraction method of typical shape defect vector for super-wide strip steel. Journal of University of Science and Technology Beijing, 2014, 36(4): 523-528. (in Chinese)

27. Y B Sun, H M Liu, Y Peng. Reduced order model for shape discrimination of strip rolling. Engineering Mechanics, 2014, 36(4): 523-528. (in Chinese)

28. J T Dai, Q D Zhang, J Qin. Analysis of local bucking for thin cold-rolled strip. Engineering Mechanics, 2011, 28(10): 236-242. (in Chinese)

29. J T Dai, Q D Zhang. Analysis and experiment on central bucking and post bucking of thin cold-rolled sheet. Chinese Journal of Mechanical Engineering, 2011, 47(2): 44-50. (in Chinese)

30. Q D Zhang, X F Lu, X F Zhang. Analysis of bucking deformation for thin cold-rolled strip with initial warping defect. Engineering Mechanics, 2014, 31(8): 243-249. (in Chinese)

31. Q D Zhang, X F Lu, X F Zhang. Deformation of warping in apparent straight strip after shearing process. Engineering Mechanics, 2014, 31(S1): 217-222. (in Chinese)

32. R Nakhoul, P Montmitonnet, M Potier. Multi-scale method for modeling thin sheet bucking under residual stresses in the context of strip rolling. International Journal of Solids and Structures, 2015, 66: 62-76.

33. D C Tran, N Tardif, H E Khaloui, et al. Thermal buckling of thin sheet related to cold rolling: latent flatness defects. Thin-Walled Structures, 2017, 113: 129-135.

34. S Abdelkhalek, H Zahrouni, N Legrand, et al. Post-bucking modeling for strips under tension and residual stresses using asymtotic numerical method. International Journal of Mechanical Science, 2015, 104: 126-137.

35. J Bai, Q D Zhang, T Z Chang, et al. Target curve setting model for automatic flatness control on stand 1 of 1420 cold tandem mill. Iron and Steel, 2007, 42(10): 56-59. (in Chinese) 
36. F Z Lu, J Wen, J Bai. Research of flatness target curve optimization for high strength steel of $2030 \mathrm{~mm}$ tandem cold mill. Steel Rolling, 2009, 26(4): 4-6. (in Chinese)

37. P F Wang, D H Zhang, J W Liu, et al. Research and application of flatness target curve setting model for cold rolling mill. Iron and Steel, 2010, 45(4): 50-55. (in Chinese)

38. M Luo. Application of flatness curve dynamic set on the hole in strip rolling process. China Metallurgy, 2015, 25(6): 49-53. (in Chinese)

39. P F Wang, Y Peng, H M Liu. Actuator efficiency adaptive flatness control model and its application in $1250 \mathrm{~mm}$ reversible cold strip mill. Journal of Iron and Steel International, 2013, 20(6): 13-20.

40. L Ma, P F Wang, D C Wang, et al. Optimization algorithm for closed-loop control system of flatness. Journal of Iron and Steel Research, 2015, 27(7): 42-45. (in Chinese)

41. H M Liu, X L Zhang, Y R Wang. Transfer matrix method of flatness control for strip mills. Journal of Materials Processing Technology, 2005, 166: 237-242.

42. H M Liu, H T He, X Y Shan, et al. Flatness control based on dynamic effective matrix for cold strip mills. Chinese Journal of Mechanical Engineering, 2009, 22(2): 287-296.

43. H M Liu, X Y Shan, C Y Jia. Theory-intelligent dynamic matrix model of flatness control for cold rolled strips. Journal of Iron and Steel Research International, 2013, 20(8): 1-7.

44. S Abdelkhalek, P Montmitonnet, N Legrand, et al. Coupled approach for flatness prediction in clod rolling of thin strip. International Journal of Mechanical Science, 2011, 53: 661-675.

45. Y K Kim, W J Kwak, T J Shin, et al. A new model for the prediction of roll force and tension profiles in flat rolling. ISIJ International, 2010, 50(11): 1644-1652.

46. B Moazeni, M Salimi. Investigations on formation of shape defects in square rolling of uniform thin flat sheet product. ISIJ International, 2013, 53(2): 257-264.

47. X H Liu, Z Y Jiang, G D Wang. Analysis of 3-D deformation for crowning strip rolling by rigid-plastic FEM. Advanced Technology of Plasticity, 1993: 717-720.

48. X C Wang, Q Yang, J W Xv, et al. Research on the improvement effect of high tension on flatness deviation in cold strip rolling. Steel Research International, 2014, 85(11): 1560-1570.

49. Y L Liu. The self-curability and explicit and implicit heritability of shape defects in cold rolling//AISTech 2015 Proceedings, Cleveland, USA, May 4-7, 2015: 2878-2893.

50. S Kapil, P Eberhard, S K Dwivedy. Dynamic analysis of coldrolling process using the finite-element method. Journal of Manufacturing Science and Engineering, 2016, 138(4): 041002.

51. J L Sun, Y Peng, H M Liu, et al. Vibration of moving strip with distributed stress in rolling process. Journal of Iron and Steel Research International, 2010, 17(4): 24-30.

52. Y Peng, Y Zhang, J L Sun, et al. Tandem strip mill's multicoupling dynamic modeling based on the thickness control. Chinese Journal of Mechanical Engineering, 2015, 28(2): 353-362.

53. D C Wang, Q L Ma, H M Liu, et al. Study off backup roll contour optimization for cold strip temper rolling mill. Iron and Steel, 2009, 44(8): 56-59. (in Chinese)

54. Z H Bai, K Wang, Y J Wang, et al. Inner roll shape optimization design technology of VC mill. China Mechanical Engineering, 2013, 24(22): 3096-3099. (in Chinese)

55. Z H Bai, L P Yang, X D Li, et al. Roll shape setting technology of hot galvanizing and planishing mill. Journal of Iron and Steel Research International, 2007, 14(1): 33-36.

56. H H Xie, A R He, J Liu, et al. Research of the roll contour optimization on $1720 \mathrm{~mm}$ cold rolling skin pass mill. China Metallurgy, 2009, 44(8): 56-59. (in Chinese)
57. G H Yang, J G Cao, J Zhang, et al. Comprehensive control for strip edge drop \& crown and flatness on tandem cold rolling mill. Metallurgical Equipment, 2008, 6: 1-4. (in Chinese)

58. G H Yang, J Zhang, J G Cao, et al. Roll configuration for edge drop control of wide strip on 4-Hi tandem cold rolling mills. Journal of Tianjin University, 2012, 45(12): 1051-1056. (in Chinese)

59. Y Peng, H M Liu, D C Wang. Simulation of type selection for 6-high cold tandem mill based on shape control ability. Journal of Central South University of Technology, 2007, 14(2): 278-284.

60. L J Xv. Flatness control in cold strip rolling and mill type selection. Beijing: Metallurgical Industry Press, 2007. (in Chinese)

61. X Q Liu. Shape simulation and mill type evaluation software development of cold rolling strip based on coupling model method. Qinhuangdao: Yanshan University, 2012. (in Chinese)

62. L Song, M G Shen, X B Chen, et al. Optimum control for technology coolant of cold rolling mill. Journal of Liaoning Technical University (Natural Science), 2014, 33(5): 647-650. (in Chinese)

63. G Y Hu, Y Cui. Optimizing the shape control process of thin strip with bright roll in cold tandem mill. Journal of Liaoning Institute of Science and Technology, 2010, 12(2): 11-12. (in Chinese)

64. S Q Li, S Q Xing, Y L Ma, et al. Study on leveling process optimization of cold rolled strip. Steel Rolling, 2011, 28(4): 16-19. (in Chinese)

65. S M Wu. Model of temper rolling for chroming base plate by double cold reduction mill. Steel Rolling, 2008, 25(4): 28-29, 42. (in Chinese)

66. H C Chen, S M Wu, X J Li. Combination control of shape and surface quality for double UCM temper mill. China Metallurgy, 2009, 19(6): 23-25. (in Chinese)

67. Z H Bai, H X Si, Q T Zhou, et al. Comprehensive optimized technology of processing lubrication system in double cold reduction. Iron and Steel, 2011, 46(6): 60-62, 73. (in Chinese)

68. Z H Bai, X D Li, J C Lian, et al. Influence of local shape wave on ribbing of cold-rolled coil. Chinese Journal of Mechanical Engineering, 2006, 42(9): 229-232. (in Chinese)

69. X D Li, H M Liu, J H Li. Origin of side convex defect for coldrolled strip coil. Journal of Iron and Steel Research, 2008, 20(7): 29-33. (in Chinese)

70. X M Zhu, G H Feng, H L Zhang. Defect analysis on ridge buckles of cold-rolled pure titanium strip. Hot Working Technology, 2014, 43(19): 227-230. (in Chinese)

71. B Yu, X L Yue, G F She, et al. Research ob roll contour optimization in solving defect of local convexity of steel coil. China Mechanical Engineering, 2008, 19(8): 969-971. (in Chinese)

72. J H Li, J Li, W P Li. Elimination of ridge defect of cold rolled strip coil. Iron and Steel, 2008, 43(9): 49-52. (in Chinese)

73. Z Y Dou, B Huang, S Q Feng. Analysis and countermeasures of bulging defect at the edge of cold-rolled products. Iron Steel Vanadium Titanium, 2012, 33(6): 91-94. (in Chinese)

74. Y L Lu, G Chen, Z Y Zhu, et al. Causes and control strategy for ridge buckle of silicon steel in continuous annealing line. Shanghai Metals, 2015, 37(2): 54-56, 62. (in Chinese)

75. X M Zhou, Q D Zhang, C S Wang, et al. Automative edge drop control system of UCMW cold mill and its optimization. Iron and Steel, 2007, 42(9): 56-59. (in Chinese)

76. Z Y Li, J R Zhu, X W Ye, et al. Development of a new edge drop control system for the PL-TCM. Baosteel Technology, 2006, 5: 40-42. (in Chinese)

77. Q Hu, X C Wang, Q Yang. Design and application of automatic edge drop control system for 6-high tandem cold rolling mill. 
Metallurgical Industry Automation, 2016, 40(1): 34-39, 44. (in Chinese)

78. K J Wang, J H Xv, S Q Li. Automatic edge drop control system of Baosteel//Proceedings of the National Production of Rolling Technology, Ningbo, China, August 14-15, 2012: 1016-1019. (in Chinese)

79. Q D Zhang, X F Zhang, J Wen. Theory and technology of transverse thickness deviation control for DI tinplate during tandem cold rolling. Journal of Mechanical Engineering, 2013, 49(24): 30-38. (in Chinese)

80. W Q Sun, Q Yang, J Shao, et al. Edge drop control technique of silicon steel for UCM tandem cold rolling mills. Journal of University of Science and Technology Beijing, 2010, 32(10): 1340-1345. (in Chinese)

81. A R He, J Shao, W Q Sun, et al. Transverse thickness deviation control of non-oriented silicon steel during cold rolling. Journal of Mechanical Engineering, 2011, 47(10): 25-30. (in Chinese)

82. R Li, Q D Zhang, X F Zhang, et al. Control method for steel strip roughness in two-stand temper mill rolling. Chinese Journal of Mechanical Engineering, 2015, 28(3): 573-579.

83. L P Xv, L You, D H Wang, et al. Application of ABB shape meter in cold-strip steel production. China Metallurgy, 2014, 24(1): 23-26. (in Chinese)

84. X G Liang, Z J Jiao, G D Wang, et al. The technology of flatness measurement in cold rolling. Metallurgical Equipment, 2006, 6: 36-39, 77. (in Chinese)

85. L J Chen, B Han, W Tan, et al. Technology status and trend of shape detecting and shape controlling of rolled strip. Steel Rolling, 2012, 29(4): 38-42. (in Chinese)

86. D Wen, Z Li. Application of non-contact shape meter in CAL of cold rolling line. Steel Rolling, 2015, 32(5): 62-65. (in Chinese)

87. G H Yang, J Zhang, J G Cao, et al. Relationship between strip amplitude and shape for shapemeter based on airflow excitation and eddy current. Transactions of Beijing Institute of Technology, 2015, 35(7): 671-676. (in Chinese)

88. H M Liu, B Q Yu, L P Yang, et al. Development of cold strip shape meter with entire roller inlayed block intelligence and its industrial application. Iron and Steel, 2011, 46(12): 86-89. (in Chinese)

89. B Q Yu, L P Yang, J L Sun. Research status of shape detecting roller of cold rolled strip. Steel Rolling, 2011, 28(2): 44-46. (in Chinese)

90. B Q Yu, L P Yang, H M Liu, et al. Development and industry application of contact shape meter with new structure. Chinese Journal of Scientific Instrument, 2010, 31(4): 904-911. (in Chinese)

91. B Q Yu, L P Yang, Z M Li, et al. Research on embedded DSP shape signal processing system for cold rolling strip. Measurement and Control Technology, 2011, 30(8): 23-26. (in Chinese)

92. J C Lian, H M Liu. Thickness and shape control for rolling strip. Beijing: Weapons Industry Press, 1996. (in Chinese)

93. V B Ginzburg. High-quantity steel rolling: theory and practice. New York: Marcel Dekker, Inc., 1993.

94. B Q Yu. Research on entire roller intelligence cold strip shape meter and its industrial application. Qinhuangdao: Yanshan University, 2010. (in Chinese)

95. H M Liu, B Q Yu, L P Yang, et al. Entire roller embedded shapemeter: China, 201310209604.2. 2015-09-16. (in Chinese)

96. H M Wu, H M Liu, B Q Yu, et al. Determination of interference fit value on entire embedded shapemeter. Journal of Central South University, 2014, 21(12): 4503-4508.

97. H M Wu. Study of thermal deformation and optimization design of entire roller embedded shapemeter roll. Qinhuangdao: Yanshan University, 2015. (in Chinese)
98. H M Wu, H M Liu, B Q Yu, et al. Transient temperature field and stress field analysis of entire roller embedded shapemeter roll. Iron and Steel, 2014, 49(5): 47-51. (in Chinese)

99. H M Liu, B Q Yu, L P Yang, et al. A type of seamless and embedded signal processor used for shapemeter: China, CN105005287A. 2015-10-28. (in Chinese)

100. L He, J Wang, F Zhang. Research and application of discrete tracking differentiator in shape flatness recognition. Journal of Iron and Steel Research, 2013, 25(2): 58-62. (in Chinese)

101. X Y Shan. Research on matrix model of shape control for cold strip mills. Qinhuangdao: Yanshan University, 2011. (in Chinese)

102. R M Li, L P Yang, B Q Yu, et al. Effect of shape detecting roll deflection on original waveform signal of cold rolling strip. Iron and Steel, 2013, 48(5): 41-45. (in Chinese)

103. R M Li, L P Yang, B Q Yu, et al. Effect of detection roll installation precision on online shape signal of cold rolling strip. Iron and Steel, 2013, 48(7): 40-40, 48. (in Chinese)

104. Z X Zhao, D C Wang, P F Wang, et al. Research and application of position error compensation model for shape meter. Iron and Steel, 2015, 50(3): 49-53. (in Chinese)

105. L P Yang, B Q Yu, R M Li, et al. Compensation model of abnormal signal and shape detection error of cold rolling strip. Chinese Journal of Mechanical Engineering, 2014, 50(6): 30-38. (in Chinese)

106. P F Wang, D H Zhang, J J Liu, et al. Research and application of the flatness measurement calculation model on cold rolling mill. Chinese Journal of Mechanical Engineering, 2011, 47(4): 58-65. (in Chinese)

107. X L He, F Wang, Z C Sun. Automatic strip shape control system of $1420 \mathrm{~mm}$ TCM at Meigang. Meishan Keji, 2010, 5: 59. (in Chinese)

108. W Q Sun, A R He, J Shao, et al. Research and application of automatic control system for high precision cold rolling. Metallurgical Industry Automation, 2015, 39(3): 44-49. (in Chinese)

109. Z F Li, H Y Li, Z H Cao, et al. Development and application of flatness control system for temper mill. Angang Technology, 2016, 1: 25-28, 62. (in Chinese)

110. D Y Gong, J Z Xv, J Zhang, et al. Shape Preset mathematic model establishment of UCM reversing mill. Steel Rolling, 2011, 28(6): 1-3. (in Chinese)

111. X G Liang. Preset model of bending force for six-high tandem cold rolling mill. Iron and Steel, 2014, 49(10): 40-43, 50. (in Chinese)

112. Z H Bai, X P Kang, S M Wu. Technology of online setting shape parameter in double frame UCM tempers. Iron and Steel, 2009, 44(5): 39-43. (in Chinese)

113. M Jelali. Performance assessment of control systems in rolling mills-application to strip thickness and flatness control. Journal of Process Control, 2007, 17: 805-816.

114. A Benporad, D Bernardini, F A Cuzzola, et al. Optimizationbased automatic flatness control in cold tandem rolling. Journal of Process Control, 2010, 20: 396-407.

115. G Pin, V Francesconi, F A Cuzzola, et al. Adaptive task-space control of strip flatness in multiroll mill stands. IFAC Proceedings Volumes, 2011, 44(1):11720-11725.

116. G Pin, V Francesconi, F A Cuzzola, et al. Adaptive task-space metal strip-flatness control in cold multi-roll mill stands. Journal of Process Control, 2013, 23: 108-119.

117. Y Zhang, Q Yang, X C Wang. Control strategies of asymmetric strip shape in six-high cold rolling mill. Journal of Iron and Steel International, 2011, 18(9): 27-32.

118. L Song, M G Shen, L P Yang, et al. Shape control dimensionality reduction efficiency inherited regulation method of cold 
rolling wide strip. Iron and Steel, 2016, 51(1): 70-75. (in Chinese)

119. X Y Shan, C Y Jia, H M Liu. Neural fuzzy PID model of tilting roll and bending roll flatness control for strip mill. Journal of Mechanical Engineering, 2009, 45(9): 254-259. (in Chinese)

120. Y N Xia, S Zhao. China Manufacturing 2025. Beijing: China Machine Press, 2016. (in Chinese)

Dong-Cheng Wang, born in 1981, is currently an associate professor at National Engineering Research Center for Equipment and Technology of Cold Rolling Strip, Yanshan University, China. He received his $\mathrm{PhD}$ degree from Yanshan University, China, in 2009.

His research interests include mechanical engineering and automation. Tel: +86-335-8387652; E-mail: wdc-731@163.com.
Hong-Min Liu, born in 1959, is currently a professor at National Engineering Research Center for Equipment and Technology of Cold Rolling Strip, Yanshan University, China. He received his $\mathrm{PhD}$ degree from Northeast Heavy Machinery Institute, China, in 1988. His research interests include three-dimensional rolling theory and numerical methods, strip shape control, rolling technology and equipment. Tel: +86-335-8057086.

Jun Liu, born in 1968, is currently a PhD candidate at Yanshan University, China. Her research interests include rolling theory and technology. 